\title{
Gastric mucosal biopsies in non ulcer dyspepsia: A histopathologic study
}

\author{
Priya Singh', K C Goswami², Bharat Bhushan Gupta ${ }^{3}$ \\ ${ }^{1}$ Department of Cytology and Gynec pathology, PGIMER, Chandigarh, ²Department of Pathology, Government Medical College, Jammu, \\ ${ }^{3}$ Postgraduate Department of Medicine, Government Medical College, Jammu
}

Background: Non-ulcer dyspepsia (NUD) is a major health problem and one of the most common causes of morbidity and economic loss. The aim of the study was to determine the pattern of gastric mucosal histopathologic alterations and frequency of Helicobacter pylori in patients with NUD. Materials and Methods: Patients presenting to Gastroenterology Out Patient Department over a period of one year with symptoms of dyspepsia but without any endoscopic lesion were taken up for the study and evaluated by obtaining two gastric mucosal biopsy specimens each from antrum and body mucosa. The specimens were processed and examined histologically using the updated Sydney system. Results: Gastric biopsies from 102 patients (63 females and 39 males) with NUD were studied. Histological examination of gastric mucosal biopsies showed that $82.3 \%$ of NUD patients had histopathological features of gastritis. Chronic inflammation was present in $84(82.3 \%)$, activity was seen in $63(61.7 \%)$, glandular atrophy was seen in $10(9.8 \%)$ and intestinal metaplasia was seen in $4(3.9 \%)$ patients with NUD on gastric biopsy. Maximum number of patients with NUD had Sydney score of less than $5(68.6 \%)$.H pylori were identified on gastric mucosal biopsies in $49(48.0 \%)$ patients with NUD based on H \& E and Geimsa stained sections. Conclusion: NUD is a clinical problem which occurs in both sexes in all age groups; prevalence being low below 20 years of age. Patients with this condition have a high frequency of gastric mucosal inflammation and $\mathrm{H}$ pylori infection.

\section{Access this article online}

Website:

http://nepjol.info/index.php/AJMS

DOI: 10.3126/ajms.v7i2.13237

E-ISSN: 2091-0576

P-ISSN: 2467-9100

Key words: Non ulcer dyspepsia, Updated sydney system, H pylori

\section{INTRODUCTION}

The term "dyspepsia" is derived from Greek words "dys" (bad) and "pepsis" (digestion) that refers to symptoms thought to originate in the upper gastrointestinal tract. Dyspepsia is often used to refer to pain or discomfort centered in the upper abdomen. ${ }^{1}$ At least $50 \%$ of patients with dyspepsia who are seen in primary care settings have non-ulcer dyspepsia in which no definite structural or biochemical explanation for the symptoms can be identified. ${ }^{2}$

A number of hypotheses have been proposed to explain the pathogenesis of non-ulcer dyspepsia which includes gastric acid hypothesis, motor-disorder hypothesis, the psychiatric hypothesis, the hypothesis of augmented visceral perception, and the food intolerance hypothesis. ${ }^{1}$ Helicobacter pylori (H pylori) infection is also probably a causative agent for nonulcer dyspepsia. ${ }^{3}$

$\mathrm{H}$ pylori is a small, curved and highly motile gram negative bacillus which survives on the surface of the mucosa in the layer of mucin and in the gastric pits. ${ }^{4}$ It is recognized to be associated with diverse upper gastrointestinal pathologies such as chronic gastritis, peptic ulceration, mucosal associated lymphoid tissue (MALT) lymphoma and gastric carcinoma. ${ }^{5,6}$ Therefore, investigation for this organism has become an integral part of upper gastrointestinal endoscopy. 
Non-ulcer dyspepsia is a major health problem and one of the most common causes of morbidity and economic loss. Patients with dyspepsia also have deterioration in the quality of their life. Hence the present study was carried out to determine the pattern of gastric mucosal histopathologic alterations in patients with non-ulcer dyspepsia and frequency of $\mathrm{H}$ pylori in these patients, which would help in better understanding and management of patients of non-ulcer dyspepsia.

\section{MATERIALS AND METHODS}

The study was conducted in the Postgraduate Department of Pathology and Gastroenterology division of the Postgraduate Department of Medicine, Government Medical College, Jammu. The study was prospective in nature, extending over a period of one year with effect from $1^{\text {st }}$ November, 2009 to $31^{\text {st }}$ October, 2010.

Patients presenting to Gastroenterology Out Patient Department over a period of one year with symptoms of dyspepsia were taken up for the study. Written consent was obtained from each patient after explaining him/ her the nature and purpose of the study. Dyspepsia was defined as the presence of one or more of the postprandial fullness, early satiation, or epigastric pain or discomfort for the last three months with symptom onset at least six months before diagnosis according to the latest Rome III criteria.

Clinical history was elicited from each patient and complete general physical and systemic examination was done. This was followed by evaluation of relevant investigations.

\section{Inclusion criteria}

1. No evidence of organic disease from the history, physical examination or laboratory tests that is likely to explain the symptoms.

2. No evidence of any mucosal lesion in the esophagus, stomach or duodenum at upper gastrointestinal (G.I.) endoscopy.

\section{Exclusion criteria}

1. Patients whose symptoms were predominantly heart burn and/or regurgitation.

2. Peptic ulcer disease.

3. Patients whose dyspepsia had been investigated previously by radiology, endoscopy or other tests and specific diagnosis established.

4. Patients who had used non steroidal anti-inflammatory drugs (NSAIDs) in the last 2 weeks prior to endoscopy.
5. Attempt at Helicobacter pylori eradication or acid suppressive therapy in the last 2 weeks prior to endoscopy.

6. Absolute/relative contra-indication to upper G.I. endoscopy.

After overnight fasting, upper gastrointestinal endoscopy was performed on selected patients using Fujinon EG-265WR Endoscope under throat anaesthesia with $10 \%$ xylocaine spray. The esophagus, stomach and duodenum were visualized and mucosal findings noticed. Patients in whom no endoscopic lesion was found in the esophagus, stomach, duodenal cap and $2^{\text {nd }}$ portion of the duodenum were further evaluated by obtaining two gastric mucosal biopsy specimens each from antrum and body mucosa. After each endoscopy, endoscopes and biopsy forceps were sterilized with $2 \%$ glutaraldehyde to prevent cross infection among patients.

The specimens were properly labeled, fixed in $10 \%$ formalin, processed using paraffin embedding technique, sectioned at 3 micrometer perpendicular to the mucosal surface and stained with haematoxylin and eosin ( $\mathrm{H} \& \mathrm{E})$. The histopathological parameters were graded using the updated Sydney system. ${ }^{7}$ Presence of $\mathrm{H}$ pylori was looked for on H\&E stained sections and one section each from antral and body mucosa was also stained by modified Geimsa stain. ${ }^{8}$

\section{RESULTS}

A total of 102 patients who presented with dyspeptic symptoms and normal upper gastrointestinal endoscopy were included in the study. They consisted of 63 females $(61.7 \%)$ and 39 males $(38.2 \%)$. Their ages ranged between 18 to 70 yrs. 31-40 year age group comprised of the maximum number of the cases which were $34.3 \%$ followed by $22.5 \%$ cases in $41-50 y e a r$ age group. Maximum number of cases both males and females were in the age group of $31-40$ years which forms $28.2 \%$ in males and $38 \%$ in females.

Out of 102 patients with non ulcer dyspepsia, $17.6 \%$ had normal gastric mucosa on histopathology and $82.3 \%$ had histopathological features of gastritis. Out of 39 male patients, gastritis was present in $32(82.0 \%)$ patients. Among 63 female patients, gastritis was seen in $52(82.5 \%)$ patients.

Chronic inflammation (Figure 1a) was present in 84 (82.3\%) patients of non- ulcer dyspepsia on gastric biopsy; out of which, 62 (73.8\%) had mild, 22 (26.1\%) had moderate 


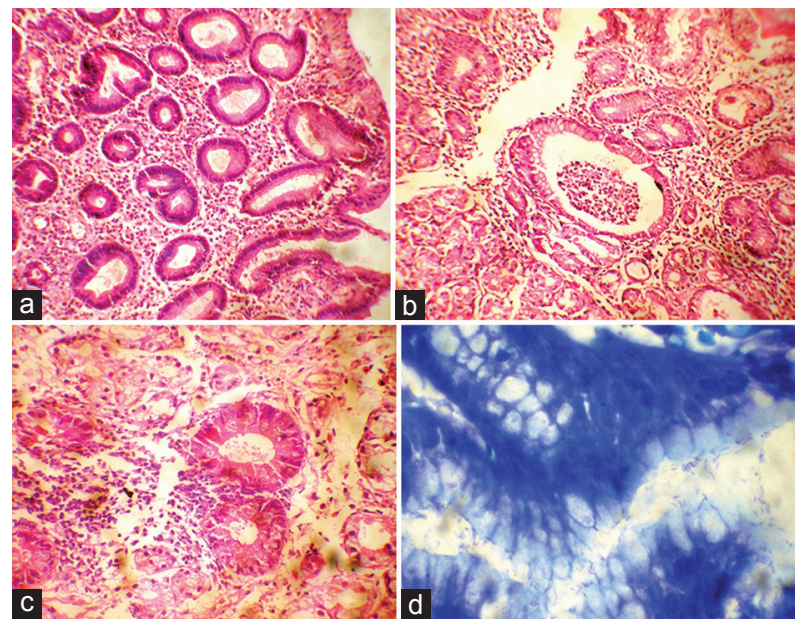

Figure 1: (a) Shows moderate chronic antral gastritis with lymphoplasmacytic infiltrate in lamina propria [H\&E, 200X], (b) Shows severely active body gastritis, note the presence of crypt abscess [H\&E, 200X], (c) Shows chronic gastritis; focal glands show intestinal metaplasia with presence of goblet cells and paneth cells [H\&E, 200X], (d) Shows numerous $\mathrm{H}$ pylori on the surface of gastric mucosa [Modified Geimsa, 100X]

and none had severe chronic inflammation. Activity (Figure 1b) was seen in $63(61.7 \%)$ patients of non- ulcer dyspepsia on gastric biopsy; out of which, $48(76.1 \%)$ had mild, $11(17.4 \%)$ had moderate and 4 (6.3\%) had severe neutrophilic activity. Glandular atrophy was seen in $10(9.8 \%)$ patients of non-ulcer dyspepsia on gastric biopsy and was of mild grade. Intestinal metaplasia (Figure 1c) was seen in $4(3.9 \%)$ patients of non-ulcer dyspepsia on gastric biopsy and was also of mild grade.

H pylori (Figure 1d) were identified on gastric mucosal biopsies in $49(48.0 \%)$ patients with non-ulcer dyspepsia; out of which, $33(67.3 \%)$ had mild, $10(20.4 \%)$ had moderate and $6(12.2 \%)$ had severe $\mathrm{H}$ pylori colonization. Lymphoid follicles and lymphoid collections were seen in $16(15.6 \%)$ patients of non-ulcer dyspepsia.

Maximum number of patients with non-ulcer dyspepsia had Sydney score of less than $5(68.6 \%) .17 .6 \%$ patients had score zero; $13.7 \%$ had score between 5 and 10 and none had score above 10.

\section{DISCUSSION}

Dyspepsia is among the most common complaints evaluated by both the general practitioner and gastroenterologist. The magnitude of non-ulcer dyspepsia is impressive and the annual incidence is over $1 \% .{ }^{9}$ The prevalence of functional dyspepsia ranges from $20 \%-30 \%$ in the general population. ${ }^{10}$ Non-ulcer dyspepsia (NUD) is not only common, but costly, because it often results in empiric treatment intended to heal peptic ulcers, including potent inhibitors of gastric acid secretion, ${ }^{11}$ and accounts for expensive tests and utilization of time. ${ }^{12}$ Altogether, dyspepsia is an important health issue and constitutes a significant clinical problem in primary care.

In our study, 31-50 years age group comprised the maximum number of the cases of non-ulcer dyspepsia $(56.8 \%)$ with peak in $4^{\text {th }}$ decade and the mean age being 42.9 yrs. Non-ulcer dyspepsia occur with a similar frequency in both sexes up to 60 years, but less common in older people of both sexes. ${ }^{13}$ In the present study, the maximum number both of male and female patients was in the age group of $31-40$ years which forms $28.2 \%$ in males and $38.0 \%$ in females. 42.9 yrs was the mean age of male and 42.3 yrs. was the mean age of female patients. Least number of cases in both male $(5.1 \%)$ and female $(1.5 \%)$ patients in our study was seen up to 20 years of age.

Biopsy sampling of the gastric mucosa at diagnostic endoscopy provides information that cannot be obtained otherwise. The most common indication for gastric biopsy is the need to know whether the patient is infected with Helicobacter pylori or not and whether the stomach shows gastritis or not. ${ }^{14}$ Some studies could not find any correlation between endoscopic or histologic gastritis and dyspeptic symptoms ${ }^{15}$ while others have reported a correlation between histological inflammation of the gastroduodenal mucosa and dyspeptic symptoms. ${ }^{16}$ Microscopic examination of gastric biopsy specimens gives, in addition to $\mathrm{H}$ pylori status, information about the grade, extent, and topography of gastritis- and atrophyrelated alterations in the gastric mucosa. This information helps in management and provides further possibilities for the assessment of risk and likelihood of various gastric disorders. ${ }^{14}$ Results from the Cochrane Collaboration systemic review shows that a one to two week course of $\mathrm{H}$. pylori eradication therapy has a small but statistically significant effect in $\mathrm{H}$ pylori positive non ulcer dyspepsia. ${ }^{17}$

In our study, histological examination of gastric mucosal biopsies showed that $82.3 \%$ had histopathological features of gastritis. This is consistent with other studies where histopathological features of gastritis was observed in the range of $70-92 \%$ in patients with NUD. ${ }^{18-20}$ Chronic gastritis alone was seen in $21(20.5 \%)$ patients, both acute and chronic gastritis in $63(61.7 \%)$ and $18(17.6 \%)$ patients had normal mucosal biopsies in the present study.

Chronic inflammation was present in $84(82.3 \%)$ patients of non ulcer dyspepsia on gastric biopsy in the present study However, a study in Nigeria observed that chronic inflammation was present in $66.7 \%$ of patients with non ulcer dyspepsia. ${ }^{21}$ Some studies have shown chronic gastritis, as characterized by an increase in round cell infiltration, in 
dyspeptic patients. ${ }^{22,23}$ The implication of gastric mucosal inflammation in patients with non ulcer dyspepsia is not clear because of lack of comparison with an appropriate control group made up of healthy subjects. ${ }^{21}$ Toukan et al. ${ }^{16}$ in their study of gastroduodenal inflammation in patients with non ulcer dyspepsia found no significant difference in the gastric mucosal round cell numbers between dyspeptic patients and normal controls.

Neutrophilic infiltration was seen in $63(61.7 \%)$ patients of non ulcer dyspepsia on gastric biopsy in our study which is in concordance to that stated by Toukan et $\mathrm{al}^{16}$ who observed a significant increase in gastroduodenal mucosal neutrophilic count in patients with non ulcer dyspepsia. In biopsy studies of normal people, few or no neutrophils are usually seen by subjective means..$^{24,25}$ Some authors see the presence of increased neutrophil infiltration as implying that the inflammatory process is active, particularly if seen in the epithelial cell layer. ${ }^{26}$ This mucosal inflammation may account for the symptoms in patients with non ulcer dyspepsia. ${ }^{16}$

Glandular atrophy was seen in $10(9.8 \%)$ patients of non ulcer dyspepsia on gastric biopsy and was of mild grade in the present study. Other studies stated atrophic gastritis in $2.5 \%{ }^{19}$ and $14.5 \% 0^{27}$ NUD patients. However, Nwokediuko \& Okafor $^{21}$ reported glandular atrophy in $42.7 \%$ patients of non ulcer dyspepsia and it was of mild grade in $28.1 \%$, moderate grade in $53.1 \%$ and severe grade in $18.7 \%$ patients. Shafii et $\mathrm{a}^{28}$ reported that patients with mild chronic gastritis are less probable to reflect atrophy which is similar to the observation made in our study. Even when extensive biopsy protocols are used, inevitable sampling errors may affect the documentation of the atrophic foci, which are frequently patchy. ${ }^{29}$

In the present study, intestinal metaplasia was seen in 4 $(3.9 \%)$ patients of non ulcer dyspepsia on gastric biopsy and was also of mild grade. This is in concordance with other studies which reported intestinal metaplasia in $2.5 \%{ }^{19}$ and $8 \%{ }^{21}$ NUD patients.

In our study, maximum number of patients with non ulcer dyspepsia had Sydney score of less than 5 (68.6\%). 17.6\% patients had score zero; $13.7 \%$ had score between 5 and 10 and none had score above 10. Nwokediuko \& Okafor $^{21}$ reported Sydney score zero in 29\%, less than 5 in 34.7\%, between 5 and 10 in $33.3 \%$ and more than 10 in $2.7 \%$ patients of non ulcer dyspepsia.

In our study, $\mathrm{H}$ pylori were identified on gastric mucosal biopsies in $49(48.0 \%)$ out of 102 patients with non ulcer dyspepsia based on $\mathrm{H} \& \mathrm{E}$ and Geimsa stained sections. Various studies show the prevalence of H pylori in NUD patients between $37.3 \%$ to $75 \% .^{30-32}$
The so called post-infection functional dyspepsia occurring after acute gastrointestinal infection postulates that inflammation may cause alterations in the enteric nervous system and visceral sensation by modifying signaling in the brain-gut axis. $\mathrm{H}$ pylori induced inflammation of the gastric mucosa in functional dyspepsia falls into the same logic, suggesting that acute and chronic inflammation seem to play some role in the functional dyspepsia pathogenesis. ${ }^{33,34}$

\section{REFERENCE}

1. Fisher RS and Parkman HP. Management of non ulcer dyspepsia. N Eng J Med 1998; 339: 1376-1381.

2. Richter JE. Dyspepsia: organic causes and differential characteristics from functional dyspepsia. Scand J Gastroenterol 1991; 26:11-16.

3. Marshall BJ. The 1995 Albert Lasker medical research award. Helicobacter pylori. The etiologic agent for peptic ulcer. JAMA 1995; 274:1064-1066.

4. Jones DM, Lessells $\mathrm{AM}$ and Eldridge J. Campylobacter like organisms on the gastric mucosa: culture, histological and serological studies. J Clin Pathol 1984; 37:1002-1006.

5. Blaser MJ. Helicobacter pylori and the pathogenesis of gastroduodenal inflammation. The Journal of Infectious diseases 1990; 161: 626-633.

6. Suerbaum S and Michetti P. Helicobacter pylori infection. N Eng J Med 2002; 347: 1175-1186.

7. Dixon MF, Genta RM, Yardley JH and Correa P. Classification and grading of gastritis. The updated Sydney system. International Workshop on the Histopathology of Gastritis, Houston 1994. Am J Surg Pathol 1996; 20: 1161 - 1181.

8. Gray SF, Wyatt JI and Rathbone BJ. Simplified techniques for identifying Campylobacter pyloridis. J Clin Pathol 1986; 39:1279-1280.

9. Bonnevie O. Outcome of non-ulcer dyspepsia. Scand J Gastroenterol 1982; 79(suppl): 135-138.

10. Hammer $\mathrm{J}$ and Talley NJ. Non ulcer dyspepsia. Curr Opin Gastroenterol 1999; 15: 492-496.

11. Cocco $A E$ and Cocco DV. A survey of cimetidine prescribing. N Engl J Med 1981; 304:1281-1284.

12. Talley NJ and Piper DW. A prospective study of social factors and major life event stress in patients with dyspepsia of unknown cause. Scand J Gastroenterol 1987; 22: 268-272.

13. Bernersen B, Johnsen $R$ and Straume B. Non-ulcer dyspepsia and peptic ulcer: the distribution in a population and their relation to risk factors. Gut 1996; 38: 822-825.

14. Elta GH, Appelman HD and Behler EM. A study of the correlation between endoscopic \& histological diagnosis in gastroduodenitis. Am J Gastroenterol 1987; 82: 749-753.

15. Jönsson KA, Gotthard R, Bodemar G and Brodin U. The Clinical Relevance of Endoscopic and Histologic Inflammation of Gastroduodenal Mucosa in Dyspepsia of Unknown Origin. Scand J Gastroenterol 1989; 24: 385-395.

16. Toukan AU, Kamal MF, Amr SS, Arnaout MA, and Abu-Romiyeh AS. Gastroduodenal inflammation in patients with nonulcer dyspepsia. A controlled endoscopic and morphometric study. Dig Dis Sci 1985; 30: 313-320.

17. Moayyedi P, Soo S, Deeks J, Delaney B, Harris A, Innes M, et al. Eradication of Helicobacter pylori for non-ulcer dyspepsia. Cochrane Database Sys Rev 2005; (1): CD002096.

18. Bechi P, Del R, Amorosi A, Marcuzzo G and Cortesini C. 
Helicobacter pylori and luminal gastric $\mathrm{pH}$. Relationships in nonulcer dyspepsia. Dig Dis Sci 1992; 37:378-384.

19. Arruda SMB, Forones NM, Juca NT and Barros KSC. Could gastric histology be a useful marker for making decision on Helicobacter pylori eradication therapy in patients with dyspepsia? Arq Gastroenterol 2009; 46: 209-213.

20. Mukhopadhyay DK, Tandon RK, Dasarathy S, Mathur M and Wali JP. A study of Helicobacter pylori in north Indian subjects with non-ulcer dyspepsia. Indian J Gastroenterol 1992; 11(3):146-147.

21. Nwokediuko SC and Okafor OC. Gastric Mucosa In Nonulcer Dyspepsia: A Histopathological Study Of Nigerian Patients. The Internet Journal of Gastroenterology 2007; 5(2).

22. Fung WP, Papadimitriou JM and Matz LR. Endoscopic, histological and ultrastructural correlations in chronic gastritis. Am J Gastroenterol 1979; 71: 269-279.

23. Tyagi KP, Mukhopadhyay AK, Aggarwal HH, Naik SR, Malik GB, Gupta DN, et al. Gastric mucosal morphology in tropics and influence of spices, tea and smoking. Nutr Metab 1974; 17: 129-135.

24. Kreuning J, Bosman FT, Kuiper G, vd Wal AM and Lindeman J. Gastric and duodenal mucosa in "healthy" individuals. An endoscopic and histopathological study of 50 volunteers. J Clin Pathol 1978; 3:69-77.

25. Mac Donald WC and Rubin CE. Gastric biopsy-a critical evaluation.Gastroenterology1967; 53:143-170.

26. Whitehead R, Truelove SC and Gera MW. The histological diagnosis of chronic gastritis in fibreoptic gastroscope biopsy specimens. J Clin Pathol 1972; 25:1-11.

27. Williams AW, Edwards F, Lewis THC and Coghill NF. Investigation of non-ulcer dyspepsia by gastric biopsy. Br Med J 1957; 16:372-377.

28. Shafii M, Nikzad SE, Kasiri H and Naghipour M. Histopathological evaluation of chronic gastritis with and without Helicobacter pylori colonization: a study from Iran. Malaysian J Pathol 2008; 30: 27-30.

29. Rugge $M$ and Genta RM. Staging and grading of chronic gastritis. Hum Pathol 2005; 36:228-233.

30. Loffeld RJLF, Potters HVPJ, Arends JW, Stobberingh E, Flendrig JA and Van Spreeuwel JP. Campylobacter associated gastritis in patients with non-ulcer dyspepsia. J Clin Pathol 1988; 4: 85-88.

31. Lee HR, Han KS, Yoo BC and Park SM. Prevalence of Helicobacter pylori Infection in Patients with Peptic Ulcer Diseases and Non-Ulcer Dyspepsia. Kor J Int Medicine 1993; 8:73-77.

32. Amin K, Alam Z, Nagra MH, Hussain I and Javed M. Association of Helicobacter pylori with non- ulcer dyspepsia. The Professional 2003; 10:1-4.

33. Spiller RC. Inflammation as a basis for functional GI disorders. Best Pract Res Clin Gastroenterol 2004; 18: 641-661.

34. Tack J, Demedts I, Dehondt G, Caenepeel P, Fischler B, Zandecki M, et al. Clinical and pathophysiological characteristics of acute-onset functional dyspepsia. Gastroenterology 2002; 122: $1738-1747$.

Authors Contribution:

PS- Concept and design of the study, collected data, reviewed the literature, manuscript preparation and critical revision of the manuscript; KCG- Concept of study, review of literature and critical revision of the manuscript; BBG- Conceptualized study, selection of cases and review of study

Source of Support: Nil, Conflict of Interest: None. 